\title{
WEAK SOLUTIONS FOR A FLUID-ELASTIC STRUCTURE INTERACTION MODEL
}

\author{
B. DESJARDINS*, M.J. ESTEBAN ${ }^{\dagger}$, \\ C. GRANDMONT ${ }^{\dagger}$ and P. LE TALLEC ${ }^{\dagger}$
}

\begin{abstract}
The purpose of this paper is to study a model coupling an incompressible viscous fluid with an elastic structure in a bounded container. We prove the existence of weak solutions "à la Leray" as long as no collisions occur.
\end{abstract}

\section{Introduction}

Because of its many applications to industrial [9], [11], [15] and biological problems [17], [18, 19], fluid-structure interaction models have been extensively studied over the past few years. Roughly speaking, the problem is to describe the evolution of an incompressible or compressible fluid coupled with a rigid or elastic structure located inside the fluid or on the domain's boundary. The purpose of this work is to prove theoretical results for the interaction between an incompressible viscous fluid governed by the Navier-Stokes equations and an elastic structure whose deformation is given by a linear combination of a finite number of modes. The case of a finite number of rigid structures embedded in a fluid was treated in [6], dealing with the incompressible NavierStokes equations as well as the compressible Navier-Stokes equations for isentropic fluids (see also [1] [5] [8] [12] [13] [20]). Following a similar approach, we intend here to prove existence of weak solutions "à la

\footnotetext{
${ }^{*}$ C.E.A., B.P. 12, 91680 Bruyères le Châtel, France

${ }^{\dagger}$ CEREMADE (UMR 7534), Université Paris-Dauphine, Place de Lattre de Tassigny, 75775 Paris cedex 16, France

2000 Mathematics Subject Classification: 35Q10, 73C02, 76D99.

Servicio de Publicaciones. Universidad Complutense. Madrid, 2001
} 
Leray" for such a fluid-elastic model. The container $\Omega$ is a $C^{2,1}$ bounded open subset of $\mathbb{R}^{d}(d=2$ or 3 ), in which an incompressible fluid of density $\bar{\rho}_{F}>0$ and viscosity $\mu>0$ evolves according to the NavierStokes equations

$$
\begin{gathered}
\bar{\rho}_{F}\left(\partial_{t} \mathbf{v}+\operatorname{div}(\mathbf{v} \otimes \mathbf{v})\right)=\operatorname{div} \underline{\underline{\sigma}}_{F}+\bar{\rho}_{F} \mathbf{f}_{F}, \text { in } \Omega_{F}(t), \\
\operatorname{div} \mathbf{v}=0 \text { in } \Omega_{F}(t), \text { and } \mathbf{v}_{\mid t=0}=\mathbf{v}_{0} \text { in } \Omega_{F}(0),
\end{gathered}
$$

where $\underline{\underline{\sigma}}_{F}=2 \mu \mathrm{D}(\mathbf{u})-p \underline{\underline{I}}$ and $\mathrm{D}(\mathbf{u})_{i j}=\left(\partial_{i} u_{j}+\partial_{j} u_{i}\right) / 2$. Here, $\mathbf{f}_{F}$ denotes bulk forces, $p$ the pressure, and $\mathbf{v}$ the Eulerian velocity field.

On the other hand, the elastic structure is described in terms of its displacement $\underline{\xi}(t, \mathbf{x})=\underline{\mathrm{X}}(t, 0, \mathbf{x})-\mathbf{x}$, where $\underline{\mathrm{X}}(t, s, \mathbf{x})$ denotes the Lagrangian flow, i.e. the position at time $t$ of the particle located at $\mathbf{x}$ at time $s$. We consider a linearized elasticity model, which writes as follows

$$
\underline{\underline{\pi}}_{1}(\underline{\xi})=\lambda \operatorname{tr}(\underline{\underline{\varepsilon}}) \underline{\underline{I}}+2 \mu \underline{\underline{\varepsilon}}, \quad \text { where } \underline{\underline{\varepsilon}}(\underline{\xi})=\frac{1}{2}\left(\nabla \underline{\xi}+{ }^{t} \nabla \underline{\xi}\right) .
$$

$\underline{\underline{\pi}}_{1}$ denotes the first Piola tensor, and $\lambda, \mu$ the Lamé constants of the elastic media satisfying $\lambda+2 \mu>0$. Denoting $\bar{\rho}_{S}(0)$ the initial density of the structure, the free dynamics would be given by $\bar{\rho}_{S}(0) \partial_{t}^{2} \underline{\xi}=\operatorname{div} \underline{\underline{\pi}}_{1}(\underline{\xi})$ in the absence of fluid. We now introduce the elastic modes in the initial elastic $C^{2,1}$ domain $\Omega_{S}(0)$

$$
-\operatorname{div} \underline{\pi}_{1}\left(\underline{\xi}_{i}\right)=\lambda_{i} \underline{\xi}_{i} \text { in } \Omega_{S}(0) \text { and } \underline{\underline{\pi}}_{1}\left(\underline{\xi}_{i}\right) \cdot \mathbf{n}_{L}=0 \text { on } \partial \Omega_{S}(0),
$$

where $\mathbf{n}_{L}$ denotes the outward normal to $\partial \Omega_{S}(0),\left\{\underline{\xi}_{i}\right\}_{i \in \mathbb{N}}$ is an orthonormal basis of $L^{2}\left(\Omega_{S}(0)\right)$ and $\left\{\lambda_{i}\right\}_{i \in \mathbb{N}}$ is a non negative non decreasing sequence of eigenvalues. The regularity of the domain $\Omega_{S}(0)$ provides us with $H^{3}$ regularity for $\underline{\xi}_{i}$. Let us also observe that the eigenvalue $\lambda_{i}=0$ corresponds to Lagrangian rigid motions $\xi(\mathbf{x})=\mathbf{a}+\mathbf{b} \times \mathbf{x}$, where $\mathbf{a} \in \mathbb{R}^{d}$ and $\mathbf{b} \in \mathbb{R}^{2 d-3}$. Given $N \geq 1$, we assume that the structure displacement is determined in terms of the first $N$ eigenmodes

$$
\underline{\xi}(t, \mathbf{x})=\underline{\xi}_{\alpha}(t, \mathbf{x}):=\sum_{i=1}^{N} \alpha_{i}(t) \underline{\xi}_{i}(\mathbf{x}), \text { where } \alpha(t)=\left\{\alpha_{i}(t)\right\}_{1 \leq i \leq N} \in \mathbb{R}^{N} .
$$

The corresponding Lagrangian flow $\mathbf{x} \mapsto \underline{X}_{\alpha}(t, 0, \mathbf{x})=\mathbf{x}+\underline{\xi}_{\alpha}(t, \mathbf{x})$ has to be a diffeomorphism from $\Omega_{S}(0)$ onto $\Omega_{S}(t):=\underline{\mathrm{X}}_{\alpha}\left(t, 0, \Omega_{S}(0)\right)$, so 
that all what follows will hold as long as $\operatorname{det} \mathbb{F}_{\alpha}(t, \mathbf{x}) \neq 0$ on $\bar{\Omega}_{s}(0)$ (where $\mathbb{F}_{\alpha}(t, \mathbf{x}):=\nabla \underline{X}_{\alpha}(t, 0, \mathbf{x})$ ), and $\underline{X}_{\alpha}$ is one-to-one on $\bar{\Omega}_{s}(0)$. Thus, we define $\underline{\mathrm{X}}_{\alpha}(0, t, \mathbf{x})$ by $\underline{\mathrm{X}}_{\alpha}(0, t,)=.\underline{\mathrm{X}}_{\alpha}(t, 0, .)^{-1}$ and $\underline{\mathrm{X}}_{\alpha}(t, s, \mathbf{x}):=$ $\underline{\mathrm{X}}_{\alpha}\left(t, 0, \underline{\mathrm{X}}_{\alpha}(0, s, \mathbf{x})\right)$. Moreover, since we consider incompressible fluid models, the fluid domain $\Omega_{F}(t)$ has constant volume, so that the volume of $\Omega_{S}(t)=\Omega \backslash \overline{\Omega_{F}(t)}$ also has to be independent of $t$, which yields the following nonlinear constraint on $\alpha$ :

$$
\int_{\Omega_{S}(0)} \operatorname{det} \mathbb{F}_{\alpha}(t, \mathbf{x}) d \mathbf{x}=\left|\Omega_{S}(0)\right|, 0 \leq t \leq T .
$$

Notice that for small enough $T$, there are functions $\alpha$ satisfying (6) for $N$ sufficiently large. Indeed, in this case, one can find $1 \leq i \leq N$ such that $\int_{\partial \Omega_{S}(0)} \xi_{i} \cdot \mathbf{n} d s \neq 0$, under which the implicit function theorem can be applied.

Thus, the evolution of $\alpha$ is given by

$$
\begin{aligned}
\int_{\Omega_{S}(0)} & \left(\left(\bar{\rho}_{S}(0) \partial_{t}^{2} \underline{\xi}_{\alpha}-\bar{\rho}_{S}(0) \mathbf{f}_{S}\right) \underline{\xi}_{i}+\underline{\pi}_{1}\left(\underline{\xi}_{\alpha}\right) \cdot \nabla \underline{\xi}_{i}\right) d x \\
= & \int_{\partial \Omega_{S}(t)}\left(\underline{\underline{\sigma}}_{F} \cdot \mathbf{n}\right) \cdot\left(\underline{\xi}_{i} \circ \underline{\mathrm{X}}_{\alpha}(t, \mathbf{x}, 0)\right) d s, \text { for all } i=1, \ldots, N
\end{aligned}
$$

$\mathbf{f}_{S}$ denoting the bulk forces in Lagrangian coordinates. In order to give a more complete description of the model, we have to precise the boundary conditions. First, because of the viscosity, the fluid sticks to the boundary: $\mathbf{v}(t, \mathbf{x})=0$ on $\partial \Omega_{F}(t) \cap \partial \Omega$, and $\mathbf{v}(t, \mathbf{x})=\partial_{t} \underline{\xi}_{\alpha}\left(t, \underline{X}_{\alpha}(0, t, \mathbf{x})\right)$ on $\partial \Omega_{F}(t) \cap \partial \Omega_{S}(t)$. Finally, note that in (7) the $N$-mode projection of the normal stress is assumed to be continuous on $\partial \Omega_{S}(t) \cap \partial \Omega_{F}(t)$ :

$$
\int_{\partial \Omega_{S}(t)}\left(\left(\underline{\underline{\sigma}}_{F}-\underline{\underline{\sigma}}_{S}\right) \cdot \mathbf{n}\right) \cdot\left(\underline{\xi}_{i} \circ \underline{\mathbf{X}}_{\alpha}(t, \mathbf{x}, 0)\right) d s=0, i=1 \ldots N,
$$

where $\underline{\underline{\sigma}}_{S}$ denotes the Cauchy stress tensor of the structure:

$$
\underline{\underline{\sigma}}_{S}\left(t, \underline{\mathrm{X}}_{\alpha}(t, 0, \cdot)\right)=\frac{\underline{\underline{\pi}}_{1}\left(\underline{\xi}_{\alpha}\right) \cdot \mathbb{F}_{\alpha}}{\operatorname{det} \mathbb{F}_{\alpha}}
$$

The aim of this paper is to define a weak formulation of the above problem (section 2) and to prove the existence of weak solutions under 
appropriate assumptions (section 4). In section 3, we describe how to represent a global Eulerian velocity compatible with (5) in terms of functions defined in fixed reference domains. Section 6 is devoted to the proof of stability for weak solutions, whereas in section 5 , we construct approximate solutions of the fluid-structure interaction problem.

Let us end the introduction with some perspectives on future work: we have by now treated the case of rigid or slightly elastic bodies embedded in a compressible or incompressible fluid. In forthcoming works, we intend to consider the case of a $2-\mathrm{D}$ or $3-\mathrm{D}$ elastic structure containing a 3 -D viscous fluid. The aforementioned situation is somehow connected to blood flow modelling $[18,19]$ which has retained more and more attention over the last few years ( $\mathrm{see}^{1}$ for numerical simulations). Other boundary conditions and solid bodies attached to the internal walls are also among the situations that we would like to tackle.

\section{Mathematical formulation}

As in [5] [6], we introduce the globally defined Eulerian velocity $\mathbf{u}$ given by $\mathbf{u}(t, \mathbf{x})=\mathbf{v}(t, \mathbf{x})$ in $\Omega_{F}(t)$ and $\mathbf{u}(t, \mathbf{x})=\partial_{t} \underline{\xi}_{\alpha}\left(t, \underline{X}_{\alpha}(0, t, \mathbf{x})\right)$ in $\Omega_{S}(t)$, and the corresponding Lagrangian flow $\underline{\mathrm{X}}$

$$
\partial_{t} \underline{\mathrm{X}}(t, s, \mathbf{x})=\mathbf{u}(t, \underline{\mathrm{X}}(t, s, \mathbf{x})), \underline{\mathrm{X}}(t, s, \mathbf{x})=\mathbf{x} \in \Omega .
$$

We also need to introduce the fluid density $\rho_{F}(t, \mathbf{x})=\bar{\rho}_{F} 1_{\Omega_{F}(t)}(\mathbf{x})$ and the total density $\rho$ which are solutions of

$$
\begin{gathered}
\partial_{t} \rho_{F}+\operatorname{div}\left(\rho_{F} \mathbf{u}\right)=0 \text { in }(0, T) \times \Omega, \quad \rho_{F \mid t=0}=\bar{\rho}_{F} 1_{\Omega_{F}(0)} \text { in } \Omega, \quad(10) \\
\partial_{t} \rho+\operatorname{div}(\rho \mathbf{u})=0 \text { in }(0, T) \times \Omega, \quad \rho_{\mid t=0}=\bar{\rho}_{F} 1_{\Omega_{F}(0)}+\bar{\rho}_{S}(0) 1_{\Omega_{S}(0)} \text { in } \Omega .
\end{gathered}
$$

Notice that the incompressibility in the fluid domain $\Omega_{F}(t)$ can be written globally as

$$
\rho_{F} \operatorname{div} \mathbf{u}=0 \text { in }(0, T) \times \Omega .
$$

In order to formulate a weak formulation for the above model, we introduce the space of test functions given by

\footnotetext{
${ }^{1}$ http://dmawww.epfl.ch/Quarteroni-Chaire/Simulations/ index.html
} 


$$
\begin{gathered}
\mathcal{V}:=\left\{\underline{\phi} \in H^{1}((0, T) \times \Omega)^{d}, \underline{\phi}(t, .) \in H_{0}^{1}(\Omega)^{d} \text { for } t \in[0, T], \rho_{F} \operatorname{div} \underline{\phi}=0\right. \\
\quad \operatorname{in}(0, T) \times \Omega, \underline{\phi}(t, \underline{\mathrm{X}}(t, 0, \mathbf{x}))=\sum_{i=1}^{N} \dot{\beta}_{i}(t) \underline{\xi}_{i}(\mathbf{x}) \\
\left.\quad \text { for } \mathbf{x} \in \Omega_{S}(0), \beta \in H^{2}(0, T)^{N}\right\}
\end{gathered}
$$

Note that for any $\phi \in \mathcal{V}$, the corresponding $\beta$ satisfies

$$
\int_{\Omega_{S}(0)}\left(\mathbb{F}_{\dot{\beta}}-\underline{\underline{I}}\right): \mathbb{F}_{\alpha}^{-1} \operatorname{det} \mathbb{F}_{\alpha} d \mathbf{x}=0
$$

Let $\Omega_{F}(0)$ and $\Omega_{S}(0)=\Omega \backslash \overline{\Omega_{F}(0)}$ be given smooth domains representing the initial fluid and elastic domain. We assume that $\delta_{0}=\mathrm{d}\left(\Omega_{S}(0), \partial \Omega\right)>$ 0 , which means that there is no contact between the structure and the container at the initial time. Let $\mathbf{v}_{0} \in L^{2}\left(\Omega_{F}(0)\right)^{d}$ such that

$$
\mathbf{v}_{0} \cdot \mathbf{n}=0 \text { on } \partial \Omega, \quad \operatorname{div} \mathbf{v}_{0}=0 \text { in } \Omega_{F}(0) .
$$

Given $\dot{\alpha}_{0} \in \mathbb{R}^{N}$ satisfying the compatibility condition

$$
\sum_{i=1}^{N} \int_{\partial \Omega_{S}(0) \cap \partial \Omega_{F}(0)} \dot{\alpha}_{i, 0} \underline{\xi}_{i} \cdot \mathbf{n}=0
$$

we define $\mathbf{u}_{0} \in L^{2}(\Omega)^{d}$ by

$$
\mathbf{u}_{0}(\mathbf{x})=\mathbf{v}_{0}(\mathbf{x}) \text { in } \Omega_{F}(0) \text { and } \mathbf{u}_{0}(\mathbf{x})=\sum_{i=1}^{N} \dot{\alpha}_{i, 0} \underline{\xi}_{i}(\mathbf{x}) \text { in } \Omega_{S}(0)
$$

The initial density $\bar{\rho}_{S}(0)$ in the elastic domain is taken in $L^{\infty}\left(\Omega_{S}(0)\right) \cap$ $H^{s_{o}}\left(\Omega_{S}(0)\right)$ for some positive $s_{o} \in(0,1)$, so that in particular $\rho_{\mid t=0} \in$ $L^{\infty}(\Omega)$.

We shall say that $\left(\rho_{F}, \alpha, \mathbf{u}\right)$ is a weak solution of the above model on $(0, T)$ if

(i) $\sqrt{\rho_{F}} \mathbf{u} \in L^{\infty}\left(0, T ; L^{2}(\Omega)\right)^{d}, \mathbf{u} \in L^{2}\left(0, T ; H_{0}^{1}(\Omega)\right)^{d}$, $\operatorname{div} \mathbf{u} \in$ $L^{\infty}((0, T) \times \Omega)$ 
(ii) There exists $\alpha \in W^{1, \infty}(0, T)^{N}, \alpha(0)=0, \dot{\alpha}(0)=\dot{\alpha}_{0}$, satisfying condition (6), and such that the Di Perna-Lions flow $\underline{\mathrm{X}}$ of $\mathbf{u}$ satisfies for a.e. $\mathbf{x} \in \Omega_{S}(0)$

$$
\underline{\mathrm{X}}(t, 0, \mathbf{x})=\mathbf{x}+\underline{\xi}_{\alpha}(t, \mathbf{x}) \text { for all } t \in(0, T) .
$$

(iii) (10), (11), and (12) hold in the sense of distributions in $(0, T) \times \Omega$.

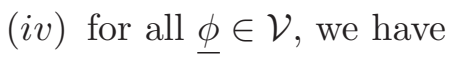

$$
\begin{gathered}
\int_{\Omega} \rho_{F} \mathbf{u} \cdot \underline{\phi}(t, \mathbf{x}) d \mathbf{x}+{ }^{t} \dot{\alpha} \cdot M_{S} \cdot \dot{\beta}(t) \\
-\int_{0}^{t} d s \int_{\Omega} d \mathbf{x}\left(\rho_{F} \mathbf{u} \cdot \partial_{t} \underline{\phi}+\rho_{F} \mathbf{u} \otimes \mathbf{u}: \mathrm{D}(\underline{\phi})\right. \\
\left.-2 \mu\left(\rho_{F} / \bar{\rho}_{F}\right) \mathrm{D}(\mathbf{u}): \mathrm{D}(\underline{\phi})+\rho_{F} \mathbf{f}_{F} \cdot \underline{\phi}\right) \\
\quad+\int_{0}^{t}\left({ }^{t} \alpha \cdot K \cdot \dot{\beta}-{ }^{t} \dot{\alpha} \cdot M_{S} \cdot \ddot{\beta}\right) d s \\
-\int_{0}^{t} d s \int_{\Omega_{S}(0)} d \mathbf{x} \bar{\rho}_{S}(0) \mathbf{f}_{S} \cdot \underline{\xi}_{\dot{\beta}}=\int_{\Omega} \rho_{F}(0) \mathbf{u}_{0} \cdot \phi_{0} d \mathbf{x}+{ }^{t} \dot{\alpha}_{0} \cdot M_{S} \cdot \dot{\beta}_{0} .
\end{gathered}
$$

Here, $K=\operatorname{diag}\left(\lambda_{1}, \ldots, \lambda_{N}\right)$, and $M_{S}$ denotes the symmetric nonnegative inertia matrix

$$
\left(M_{S}\right)_{i j}:=\int_{\Omega_{S}(0)} \bar{\rho}_{S}(0) \underline{\xi}_{i}(\mathbf{x}) \cdot \underline{\xi}_{j}(\mathbf{x}) d \mathbf{x} .
$$

Notice that $M_{S}$ is positive definite as soon as $\bar{\rho}_{S}(0)$ is positive and bounded away from zero, which is an assumption that we make from now on.

Taking $\underline{\phi}=\mathbf{u}$ as a test function in (16), we obtain the a priori energy estimate:

$$
\begin{array}{r}
\left\{\int_{\Omega} \rho_{F} \frac{\mathbf{u}^{2}}{2} d \mathbf{x}+\frac{{ }^{t} \dot{\alpha} \cdot M_{S} \cdot \dot{\alpha}}{2}+\frac{{ }^{t} \alpha \cdot K \cdot \alpha}{2}\right\}(t) \\
+\int_{0}^{t} d s \int_{\Omega} d \mathbf{x}\left(2 \mu \frac{\rho_{F}}{\bar{\rho}_{F}} \mathrm{D}(\mathbf{u}): \mathrm{D}(\mathbf{u})\right) \\
\leq \int_{0}^{t} \int_{\Omega} \rho_{F} \mathbf{f}_{F} \cdot \mathbf{u} d \mathbf{x} d s+\int_{0}^{t} \int_{\Omega_{S}(0)} \bar{\rho}_{S}(0) \mathbf{f}_{S} \cdot \underline{\xi}_{\dot{\alpha}} d \mathbf{x} d s
\end{array}
$$




$$
+\int_{\Omega} \rho_{F} \frac{\mathbf{u}_{0}^{2}}{2} d \mathbf{x}+\frac{{ }^{t} \dot{\alpha}_{0} \cdot M_{S} \cdot \dot{\alpha}_{0}}{2}
$$

Thus, as soon as $\mathbf{u}_{0} \in L^{2}(\Omega)^{d}, \mathbf{f} \in L^{2}((0, T) \times \Omega)^{d}$, we have $\sqrt{\rho_{F}} \mathbf{u} \in$ $L^{\infty}\left(0, T ; L^{2}(\Omega)\right)^{d}, \alpha \in W^{1, \infty}(0, T)^{N}$ and $\mathbf{u} \in L^{2}\left(0, T ; H_{0}^{1}(\Omega)\right)^{d}$.

\section{Representation of velocities}

In this section, we introduce an appropriate representation for admissible velocities $\mathbf{u}$, which will allow to build approximate solutions for $(i)$ and $(i i)$, and to regularize velocity fields in a suitable way. The goal here is to represent any global Eulerian velocity $\mathbf{u}$ such that $\mathbf{u}_{\mid \Omega_{F}(t)}$ is incompressible and $\mathbf{u}_{\mid \Omega_{S}(t)}$ is given by (5) by functions defined in fixed reference domains. This allows to use a fixed point approach without directly dealing with the domain variation. Let to be the set

$\left\{\widetilde{\mathbf{u}} \in L^{\infty}\left(0, T ; L^{2}\left(\Omega_{F}(0)\right)\right)^{d} \cap L^{2}\left(0, T ; H_{0}^{1}\left(\Omega_{F}(0)\right)\right)^{d}, \operatorname{div} \widetilde{\mathbf{u}}=0\right.$ in $\left.\Omega_{F}(0)\right\}$,

endowed with the natural associated norm $|\cdot|_{Y_{0}}$. The elastic domain configurations are represented by

$$
Y_{1}:=\left\{\alpha \in W^{1, \infty}(0, T)^{N}, \int_{\Omega_{S}(0)} \operatorname{det} \mathbb{F}_{\alpha}(t, \mathbf{x}) d \mathbf{x}=\left|\Omega_{S}(0)\right|\right\},
$$

endowed with the corresponding ambiant norm. The natural norm on the product space $Y_{[0, T]}=Y_{0} \times Y_{1}$ is then given by $\|(\widetilde{\mathbf{u}}, \alpha)\|_{Y_{[0, T]}}=$ $|\widetilde{\mathbf{u}}|_{Y_{0}}+|\alpha|_{Y_{1}}$. As noted in section $1, Y_{1} \neq \emptyset$ if $N$ is sufficiently large.

Let $\widetilde{\mathbf{u}} \in Y_{0}$ be given. Extending $\widetilde{\mathbf{u}}$ by 0 outside $\Omega_{F}(0)$, we deduce from Di Perna-Lions theory [7] the existence of a unique generalized flow $\underline{\widetilde{X}} \in L^{\infty}((0, T) \times \Omega)$ such that

- For all $\beta \in C^{0,1}(\mathbb{R} ; \mathbb{R})$, we have

$$
\partial_{t} \beta(\tilde{X})=\widetilde{\mathbf{u}}(t, \underline{\tilde{X}}(t, s, \mathbf{x})) \cdot \mathrm{D} \beta(\widetilde{X}) \text {, in } \mathcal{D}^{\prime}\left((0, T)^{2} \times \Omega\right) .
$$

- For a.e. $\mathbf{x} \in \Omega_{F}(0)$, for all $\left(t_{1}, t_{2}, t_{3}\right) \in(0, T)^{3}$,

$$
\underline{\tilde{X}}\left(t_{1}, t_{2}, \mathbf{x}\right)=\underline{\tilde{X}}\left(t_{1}, t_{3}, \underline{\tilde{X}}\left(t_{3}, t_{2}, \mathbf{x}\right)\right),
$$


- Denoting $\lambda^{0}$ the Lebesgue measure on $\Omega_{F}(0)$, we have

$$
\lambda^{0} \circ \underline{\tilde{\mathrm{X}}}(t, s, .)=\lambda^{0} .
$$

A natural extension of $\underline{\widetilde{\mathrm{X}}}$ to the whole domain $\Omega$ is given by $\underline{\widetilde{\mathrm{X}}}(t, s, \mathbf{x})=\mathbf{x}$ for $\mathbf{x} \in \Omega_{S}(0),(t, s) \in(0, T)^{2}$.

Given $\alpha \in Y_{1}$, we build $\underline{X}_{E}$ defined on $(0, T)^{2} \times \Omega_{S}(0)$ by

$$
\underline{\mathrm{X}}_{E}(t, 0, \mathbf{x})=\mathbf{x}+\underline{\xi}_{\alpha}(t, \mathbf{x})=\mathbf{x}+\sum_{i=1}^{N} \alpha_{i}(t) \underline{\xi}_{i}(\mathbf{x}),
$$

$$
\underline{\mathrm{X}}_{E}(0, t, .):=\underline{\mathrm{X}}_{E}(t, 0, .)^{-1} \text { and } \underline{\mathrm{X}}_{E}(t, s, \mathbf{x}):=\underline{\mathrm{X}}_{E}\left(t, 0, \underline{\mathrm{X}}_{E}(0, s, \mathbf{x})\right),
$$

which is smooth in $\mathbf{x}$ and Lipschitz in $t$ and $s$. Assuming that $\mathrm{d}\left(\Omega_{S}(t), \partial \Omega\right) \geq \delta_{1}>0$ on $(0, T)$, where $\Omega_{S}(t)=\underline{\mathrm{X}}_{E}\left(t, 0, \Omega_{S}(0)\right)$, we want to build an extension $\underline{\hat{X}}_{E}$ of $\underline{X}_{E}$ in $(0, T)^{2} \times \Omega$ which preserves the Lebesgue measure on $\Omega_{F}(0)$. First, we define the Eulerian velocity $\mathbf{u}_{E}$ of $\underline{\mathrm{X}}_{E}$ by $\mathbf{u}_{E}(t, \mathbf{x})=\partial_{t} \underline{\mathrm{X}}_{E}\left(t, 0, X_{E}(0, t, \mathbf{x})\right)$ for $\mathbf{x} \in \Omega_{S}(t)$. Next, for a fixed time $t \in(0, T)$, we extend it on $\Omega$ with $\widehat{\mathbf{u}}_{E}$ defined by solving a Stokes problem

$$
\begin{gathered}
-\mu \Delta \widehat{\mathbf{u}}_{E}+\nabla \widehat{p}_{E}=0, \operatorname{div} \widehat{\mathbf{u}}_{E}=0, \text { in } \Omega_{F}(t), \\
\widehat{\mathbf{u}}_{E}=0 \text { on } \partial \Omega \cap \partial \Omega_{F}(t), \widehat{\mathbf{u}}_{E}=\mathbf{u}_{E} \text { on } \partial \Omega_{F}(t) \cap \partial \Omega_{S}(t) .
\end{gathered}
$$

We claim that the corresponding vector field $\widehat{\mathbf{u}}_{E}$ extended by $\mathbf{u}_{E}$ on $\Omega_{S}(t)$ belongs to $L^{\infty}\left(0, T ; W^{1, \infty}(\Omega)\right)^{d}$. Indeed, this is a consequence of the regularity properties of $\mathbf{u}_{E}$ combined with classical results for the Stokes problem. The associated Lagrangian flow $\widehat{\mathrm{X}}_{E}$ thus belongs to $W^{1, \infty}((0, T) \times \Omega)^{d}$.

Finally, we introduce $\underline{\mathrm{X}}$ defined for a.e. $\mathbf{x}$ in $(0, T)^{2} \times \Omega$ by

$$
\underline{\mathrm{X}}(t, 0, \mathbf{x})=\underline{\widehat{\mathrm{X}}}_{E}(t, 0, \underline{\tilde{\mathrm{X}}}(t, 0, \mathbf{x})) .
$$

In some sense, $\underline{\widehat{X}}_{E}$ is a renormalization of $\underline{\widetilde{X}}$ depending smoothly on t. Moreover, if $A \subset \Omega_{F}(0),|\underline{\widetilde{X}}(t, 0, A)|=|\bar{A}|$ and $\underline{\widetilde{X}}(t, 0, A) \subset \Omega_{F}(0)$. Hence, $\left|\underline{\widehat{X}}_{E}(t, 0, \underline{\widetilde{X}}(t, 0, A))\right|=|\underline{\widetilde{X}}(t, 0, A)|=|A|$. It follows that $\underline{\mathrm{X}}$ preserves the volume of subsets of $\Omega_{F}(0)$. Note also that $\underline{X}(t, 0, \cdot)$ coincides 
with $\underline{\mathrm{X}}_{E}(t, 0, \cdot)$ in $\Omega_{S}(0)$. Notice that the Eulerian velocity associated with $\underline{\mathrm{X}}$ is divergence-free in $\Omega_{F}(t)$. In conclusion, given $(\tilde{\mathbf{u}}, \alpha) \in Y_{[0, T]}$ we construct a velocity $\mathbf{u}$ satisfying $(i)$ and (ii) (and reciprocally).

Finally, let us call $\mathbf{u}=\Theta(\widetilde{\mathbf{u}}, \alpha)$ the Eulerian velocity associated with $\underline{\mathrm{X}}$. Notice that this representation procedure allows us to regularize Eulerian velocities satisfying $(i)$ and (ii), by first regularizing separately the two components of $\Theta^{-1}(\mathbf{u}), \widetilde{\mathbf{u}}$ and $\alpha$, and then constructing a new regularized velocity by taking the image by $\Theta$ of the regularized $\widetilde{\mathbf{u}}$ and $\alpha$.

\section{Main result}

The aim of this paper is to prove existence of weak solutions "à la Leray", i.e. solutions satisfying the bounds associated with the energy inequality (17). Let us mention at this point the limitations of our result, which as in [5] [6] [1] (in the rigid case) holds away from collisions. In the elastic case, we are not only limited to times $t$ such that $\delta(t)=\mathrm{d}\left(\Omega_{S}(t), \partial \Omega\right)>0$, but also by the fact that $\mathbf{x} \mapsto \underline{\mathbf{X}}_{\alpha}(t, 0, \mathbf{x})$ has to be a diffeomorphism from $\bar{\Omega}_{S}(0)$ onto $\bar{\Omega}_{S}(t)$, which constrains the choice of the elastic model. Hence we have to restrict ourselves to times $t$ such that $\gamma(t)=\inf _{\mathbf{x} \in \overline{\Omega_{S}(0)}}\left|\operatorname{det} \mathbb{F}_{\alpha}(t, \mathbf{x})\right|>0$, and $\mathbf{x} \mapsto \underline{\mathbf{X}}_{\alpha}(t, 0, \mathbf{x})$ in one-to-one on $\bar{\Omega}_{S}(0)$. Also, we have to ensure that $Y_{1}$ is non empty, which writes as

$$
\kappa(t)=\min _{1 \leq i \leq N}\left|\int_{\partial \Omega_{S}(0)} \operatorname{det} \mathbb{F}_{\alpha}\left(\mathbb{F}_{\alpha}^{-1} \cdot n\right) \cdot \underline{\xi}_{i} d s\right|>0
$$

Theorem 4.1. Under assumption (14) on the initial data, and if $\delta(0)>$ $0, \kappa(0)>0, \bar{\rho}_{S}(0) \geq c_{0}>0$ in $\Omega_{S}(0)$ for some $c_{0}$, there exists $T^{*} \in$ $(0, \infty]$

$$
T^{*}=\sup \left\{t>0, \delta(t) \gamma(t) \kappa(t)>0, \underline{X}_{E}(t, 0, \cdot) \text { is one-to-one on } \overline{\Omega_{S}(0)}\right\}
$$

and a weak solution $\left(\rho, \rho_{F}, \mathbf{u}\right)$ to the above system such that the energy inequality (17) holds for a.e. $t \in\left(0, T^{*}\right)$. 
In order to prove Theorem 4.1, we consider a sequence $\left(\rho^{n}, \rho_{F}^{n}, \mathbf{u}^{n}\right)$ of approximate solutions - which we will actually build in section 5 corresponding to regularized data $\mathbf{u}_{0}^{n}$ converging strongly to the limit ones. Then, using uniform energy bounds with respect to $n$, we prove that up to the extraction of a subsequence, $\rho^{n}$ and $\rho_{F}^{n}$ converge strongly in $C\left([0, T] ; L^{p}(\Omega)\right)$ for all $p<\infty$ to some $\rho$ and $\rho_{F}$, and $\mathbf{u}^{n}$ converge to $\mathbf{u}$ in $L^{2}((0, T) \times \Omega)^{d}$. Finally, we have to check that (16) holds for any given $\underline{\phi} \in \mathcal{V}$, which is done by approximating $\underline{\phi}$ by $\underline{\phi}_{n} \in \mathcal{V}_{n}$, testfunctions adapted to $\rho_{F}^{n}$ and $\mathbf{u}^{n}$. Let us emphasize that this methodology was originated in the work of Tartar [22] for conservation laws, and later by Di Perna and Lions for transport equations [7].

\section{Construction of approximate solutions}

In order to prove Theorem 4.1, we proceed along the lines of [6] to obtain existence results for suitable approximate problems $\left(P_{\varepsilon}\right)$. These problems are easier to deal with than the original one, because the involved convecting velocities are suitably regularized. Given $T>0$ small enough to ensure

$$
\min (\delta(t), \gamma(t), \kappa(t)) \geq \alpha_{0}>0 \text { on }(0, T),
$$

which can be achieved using the space and time Lipschitz a priori bounds on the solid dynamics (see (17)), we introduce

$$
E(T)=C_{0}\left(\left|\mathbf{u}_{0}\right|_{L^{2}(\Omega)}^{2}+\int_{0}^{T}|\mathbf{f}|_{L^{2}(\Omega)}^{2} d s\right),
$$

which controls the energy in the interval $(0, T)$ in view of the a priori bound (17).

In order to define $\left(P_{\varepsilon}\right)$, we first introduce two smoothing operators $R_{\varepsilon}^{1} R_{\varepsilon}^{2}$ acting respectively on functions in $Y_{0}$ and $Y_{1}$ with values in the space of analytic functions in time. Let $\bar{\alpha} \in Y_{1}$. Since $R_{\varepsilon}^{2}(\bar{\alpha})$ has to satisfy (6), one first has to regularize $N-1$ modes $\left\{\alpha_{j}\right\}_{j \neq j_{0}}$, where $j_{0}$ is such that

$$
\int_{\partial \Omega_{S}(0)} \underline{\xi}_{j} \cdot \mathbf{n} d s \neq 0 .
$$

Then, the remaining mode is determined by the use of the implicit function theorem. Finally, we define $R_{\varepsilon}:=\left(R_{\varepsilon}^{1}, R_{\varepsilon}^{2}\right)$. 
Let $B_{T^{\prime}}\left(0 \leq T^{\prime} \leq T\right)$ be the ball of $Y_{\left[0, T^{\prime}\right]}$ centered in 0 of radius $R_{T^{\prime}}=2 E\left(T^{\prime}\right)$. Let us now take $\mathbf{w}=(\overline{\mathbf{u}}, \bar{\alpha}) \in B_{T}$. For $\varepsilon>0$ given, we split $(0, T)$ into $M$ subintervals $I_{k}=\left[t_{k}, t_{k+1}\right), 0 \leq k \leq M-1$, $t_{k}=k T / M$ such that

$$
\sup _{t \in I_{k}}\left|\nabla_{\mathbf{x}} X_{\mathbf{w}}^{\varepsilon}\left(t, t_{k}, .\right)-I\right|_{L^{\infty}(\Omega)} \leq \frac{1}{2},
$$

$X_{\mathbf{w}}^{\varepsilon}$ denoting the Lagrangian flow associated with $\Theta\left(R_{\varepsilon}(\mathbf{w})\right)$. Notice that $M$ depends only on $\varepsilon$ and on $R_{T}$.

Now, on the interval $I_{1}=\left(0, t_{1}\right)$ we build $\mathbf{u}_{\varepsilon}=F_{\varepsilon}(\overline{\mathbf{u}}, \bar{\alpha})$ as the unique solution of the linear problem in $I_{1}$ which is obtained by smoothing out the initial velocity $\mathbf{u}_{0}$, by replacing $\mathbf{u}$ by $\mathbf{v}_{\varepsilon}:=\Theta\left(R_{\varepsilon}(\mathbf{w})\right)$ in (10) and (11). This yields density functions $\rho_{F}^{\varepsilon}$ and $\rho^{\varepsilon}$, which define a set of test functions $\mathcal{V}_{\varepsilon}$. Then, $\mathbf{u}_{\varepsilon}$ will be the function solving $(i)$ to $(i v)$, with $\rho_{F}$ replaced by $\rho_{F}^{\varepsilon}$ in $(i)$ and (iv); $\alpha$ replaced by $R_{\varepsilon}^{2}(\bar{\alpha})$ in $(i i)$ and $(i v), \mathcal{V}$ replaced by $\mathcal{V}_{\varepsilon}$ in $(i v)$ and $\mathbf{u} \otimes \mathbf{u}$ by $\mathbf{v}_{\varepsilon} \otimes \mathbf{u}$ in (16).

The procedure to show that such a $\mathbf{v}_{\varepsilon}$ exists is the same as that used in [6] (Galerkin approximation method on the Lagrangian formulation). Then we show that $F_{\varepsilon}$ is compact by using classical parabolic regularity results. We basically prove that $\sqrt{\rho_{F}^{\varepsilon}} \mathbf{u}_{\varepsilon}$ belongs to $L^{\infty}\left(0, t_{1} ; H^{1}\left(\Omega_{F}(0)\right)\right.$, $\mathbf{u}_{\varepsilon}$ to $L^{2}\left(0, t_{1} ; H^{2}\left(\Omega_{F}(0)\right)\right), \partial_{t} \mathbf{u}_{\varepsilon} \in L^{2}\left(\left(0, t_{1}\right) \times \Omega_{F}(0)\right)$, and $\alpha_{\varepsilon}=R_{\varepsilon}(\bar{\alpha}) \in$ $H^{2}\left(0, t_{1}\right)^{N}$. This yields the existence of a fixed point for $F_{\varepsilon}$ in view of Leray-Schauder Theorem. We proceed similarly on $I_{2}$, starting from $\mathbf{u}\left(t_{1},.\right) \in H^{1}\left(\Omega_{F}(0)\right)$ and using the energy a priori bound (17) from $t_{1}$ to $t_{2}$. This provides us with a sequence of approximate solutions on $(0, T)$ which as in [6] satisfy energy bounds uniform in $\varepsilon$.

\section{Compactness results}

Let $\left(\rho^{n}, \mathbf{u}^{n}\right)$ be a sequence of approximate solutions with initial data $\rho_{0}^{n}$ and $\mathbf{u}_{0}^{n}$ converging to $\rho_{0}$ and $\mathbf{u}_{0}$ in $L^{1}(\Omega)$ and $L^{2}(\Omega)^{d}$ respectively, and such that the energy bounds (17) hold uniformly in $n$. As a result, the corresponding sequence $\alpha_{n}$ is bounded in $W^{1, \infty}(0, T)^{N}$ uniformly in $n$. Let us observe that

$\left|\mathbb{F}_{\alpha_{n}}(t, .)-\mathbb{F}_{\alpha_{n}}(s, .)\right|_{L^{\infty}\left(\Omega_{S}(0)\right)} \leq C\left|\alpha_{n}(t)-\alpha_{n}(s)\right| \leq C\left|\alpha_{n}\right|_{W^{1, \infty}(0, T)}|t-s|$, 


$$
\left|\nabla \mathbb{F}_{\alpha_{n}}\right|_{L^{\infty}\left((0, T) \times \Omega_{S}(0)\right)} \leq C\left|\alpha_{n}\right|_{L^{\infty}(0, T)} \leq C T,
$$

and recall that there exists $a_{0}$ and $b_{0}$ such that if $M \in \mathcal{M}_{d}(\mathbb{R})$ satisfies $\|M\|_{\infty} \leq a_{0}$, then $\operatorname{det}(I+M) \geq b_{0}>0$. Taking $s=0$ in (24) and $T_{0}$ small enough with respect to $a_{0}$, we deduce that

$$
\gamma_{n}(t) \geq \gamma_{1}>0 \text { and } \delta_{n}(t) \geq \delta_{1}>0 \text { on }\left[0, T_{0}\right]
$$

and $\quad \mathbf{x} \mapsto \underline{\mathrm{X}}_{\alpha}(t, 0, \mathbf{x}) \quad$ in one-to-one on $\bar{\Omega}_{S}(0)$ for $t \in\left[0, T_{0}\right]$. Moreover, using the particular expression of $\mathbf{u}^{n}$ in $\Omega_{S}(t)$, we deduce that $\mathbf{u}^{n}$ is bounded in $L^{\infty}\left(0, T_{0} ; L^{2}(\Omega)\right)^{d} \cap L^{2}\left(0, T_{0} ; H_{0}^{1}(\Omega)\right)^{d}$, and $\operatorname{div} \mathbf{u}^{n}$ is bounded in $L^{\infty}\left(\left(0, T_{0}\right) \times \Omega\right)$, uniformly in $n$.

First, using Ascoli's theorem, we may assume, up to the extraction of a subsequence, that $\alpha_{n}$ converges to some $\alpha \in W^{1, \infty}\left(0, T_{0}\right)^{N}$ in $C^{0}\left(\left[0, T_{0}\right]\right)^{N}$, and $\mathbb{F}_{\alpha_{n}}$ converges to $\mathbb{F}_{\alpha}$ in $C^{0}\left(\left[0, T_{0}\right] \times \overline{\Omega_{S}(0)}\right)$, so that $\operatorname{det} \mathbb{F}_{\alpha_{n}}$ converges to det $\mathbb{F}_{\alpha}$ in $C^{0}\left(\left[0, T_{0}\right] \times \overline{\Omega_{S}(0)}\right)$. Di Perna-Lions compactness results [7] immediately yield the convergence of $\rho_{F}^{n}$ to some $\rho_{F}$ in $C\left(\left[0, T_{0}\right] ; L^{p}(\Omega)\right)$ for all $p<\infty$. On the other hand, $\rho^{n}$ converges strongly to $\rho$ in $L^{p}\left(\left(0, T_{0}\right) \times \Omega\right)$ for all $p<\infty$; indeed, $\rho_{S}^{n}:=\rho^{n}-\rho_{F}^{n}$ is solution of $\partial_{t} \rho_{S}^{n}+\operatorname{div}\left(\rho_{S}^{n} \mathbf{u}^{n}\right)=0, \rho_{S \mid t=0}^{n}=\bar{\rho}_{S}(0) 1_{\Omega_{S}(0)}$. Since $\mathbf{u}_{\mid \Omega_{S}(t)}^{n}=\mathbf{u}_{E}^{n}$, we remark that $\partial_{t} \rho_{S}^{n}+\operatorname{div}\left(\rho_{S}^{n} \widehat{\mathbf{u}}_{E}^{n}\right)=0$, where the vector field $\widehat{\mathbf{u}}_{E}^{n}$ is given by the Stokes' extension (19). Since $\widehat{\mathbf{u}}_{E}^{n}$ is uniformly bounded in $L^{\infty}\left(0, T ; W^{1, \infty}(\Omega)\right)^{d}, \rho_{S}^{n}$ is bounded in $L^{\infty}\left(0, T ; H^{s_{o}}(\Omega)\right)$ for some $s_{o} \in(0,1)($ see $[3])$.

$$
\left|\rho_{S}^{n}\right|_{L^{\infty}\left(0, T ; H^{s_{o}}(\Omega)\right)} \leq C\left|\rho_{S}(0)\right|_{H^{s_{o}(\Omega)}} \exp \left(C \int_{0}^{T}\left|\nabla \widehat{\mathbf{u}}_{E}^{n}\right|_{L^{\infty}(\Omega)} d s\right)
$$

where $\rho_{S}(0)$ is an extension of $\bar{\rho}_{S}(0)$ in $H^{s_{o}}(\Omega)$. Classical compactness results yield the claimed convergence.

Next, we claim that

Proposition 1. Let $T>0$ such that $\delta(t) \geq \delta_{1}>0$ and $\gamma(t) \geq \gamma_{1}>0$ for $t \in(0, T)$. Then, for all $h>0$ small enough, we have

$$
\sup _{n \in \mathbb{N}} \int_{0}^{T} \int_{\Omega} \rho^{n}\left|\mathbf{u}_{+}^{n}-\mathbf{u}^{n}\right|^{2} d t d \mathbf{x} \leq C_{\delta_{1}, \gamma_{1}, T} h^{\alpha_{*}},
$$

where $\alpha_{*}=2 /(d+2)$ and $g_{+}(t, \mathbf{x}):=g(t+h, \mathbf{x})$ for $g \in \mathcal{D}^{\prime}(t, \mathbf{x})$. 
Proof. as in [2], [6], we denote by $A^{n}$ the argument of the left hand side of (27), and make the following splitting

$$
\begin{aligned}
& A^{n} \leq \int_{0}^{T} \int_{\Omega}\left(\rho^{n}+\rho_{+}^{n}\right)\left|\mathbf{u}_{+}^{n}-\mathbf{u}^{n}\right|^{2} d t d \mathbf{x}=A_{1}^{n}+2 A_{2}^{n}, \\
& \text { where } A_{1}^{n}=\int_{0}^{T} \int_{\Omega}\left(\rho_{+}^{n}-\rho^{n}\right)\left(\left|\mathbf{u}^{n}\right|^{2}-\left|\mathbf{u}_{+}^{n}\right|^{2}\right) d t d \mathbf{x}, \\
& \text { and } A_{2}^{n}=\int_{0}^{T} \int_{\Omega}\left(\rho_{+}^{n} \mathbf{u}_{+}^{n}-\rho^{n} \mathbf{u}^{n}\right) \cdot\left(\mathbf{u}_{+}^{n}-\mathbf{u}^{n}\right) d t d \mathbf{x} .
\end{aligned}
$$

$A_{1}^{n}$ is handled using the transport equation satisfied by $\rho^{n},(11)$. Indeed, $H_{\eta}$ being a smoothing kernel such that

$$
\left\|u-H_{\eta} u\right\|_{L^{2}} \leq C \eta\|\nabla u\|_{L^{2}} \text { (see [4] for possible choices of } H_{\eta} \text { ), }
$$

we have:

$$
\begin{aligned}
& \begin{array}{l}
A_{1}^{n} \leq \int_{0}^{T} \int_{\Omega}\left(\rho_{+}^{n}-\rho^{n}\right)\left(\left|H_{\eta} \mathbf{u}^{n}\right|^{2}-\left|H_{\eta} \mathbf{u}_{+}^{n}\right|^{2}\right) d t d \mathbf{x} \\
+\eta^{2}\left|\rho_{+}^{n}-\rho^{n}\right|_{L^{\infty}((0, T) \times \Omega)}\left|\nabla \mathbf{u}^{n}\right|_{L^{2}\left(0, T+h ; L^{2}(\Omega)\right)}^{2}
\end{array} \\
& \leq C \eta^{2}+ \\
& \left|\int_{0}^{T} d t \int_{t}^{t+h} d s \int_{\Omega} d \mathbf{x} \rho^{n}(s, \mathbf{x}) \mathbf{u}_{i}^{n}(s, \mathbf{x}) \cdot\left(\nabla^{i} H_{\eta} \mathbf{u}_{+}^{n} \cdot H_{\eta} \mathbf{u}_{+}^{n}-\nabla^{i} H_{\eta} \mathbf{u}^{n} \cdot H_{\eta} \mathbf{u}^{n}\right)\right| \\
& \leq C \eta^{2}+ \\
& C h\left|\rho^{n} \mathbf{u}^{n}\right|_{L^{\infty}\left(0, T+h ; L^{2}(\Omega)\right)}\left|\nabla H_{\eta} \mathbf{u}^{n}\right|_{L^{2}((0, T+h) \times \Omega)}\left|H_{\eta} \mathbf{u}^{n}\right|_{L^{\infty}((0, T+h) \times \Omega)} \\
& \leq C \eta^{2}+C h \eta^{-d / 2} .
\end{aligned}
$$

In order to estimate $A_{2}^{n}$, we will use the weak formulation (16) with $\mathbf{w}^{n}:=\mathbf{u}_{+}^{n}-\mathbf{u}^{n}$ as a test function in $[t, t+h]$. However, $\mathbf{w}^{n}$ has to be slightly modified in order to be admissible. Observing that $\mathbf{u}^{n}=$ $\widehat{\mathbf{u}}_{E}^{n}+\left(\mathbf{u}^{n}-\widehat{\mathbf{u}}_{E}^{n}\right)$, and that $\mathbf{u}_{2}^{n}:=\mathbf{u}^{n}-\widehat{\mathbf{u}}_{E}^{n}$ vanishes in $\Omega_{S}(t)$ and thus is incompressible in $\Omega$, we slightly modify $\mathbf{u}_{2}^{n}$ as follows: we first use the fact that $\delta(t) \geq \delta_{1}>0$ and the fact that $\underline{\mathrm{X}}_{\alpha}$ is one-to-one on $\bar{\Omega}_{s}(0)$ to split $\mathbf{u}_{2}^{n}$ as in [6] into a velocity field $\mathbf{u}_{2,1}^{n}$ supported away from $\Omega_{S}(t)$, and $\mathbf{u}_{2,2}^{n}$ supported away from the boundary. Then, we make a dilation centered in $\Omega_{S}(t)$ of magnitude $\eta>>h$, so that $\mathbf{u}_{2,2, \eta}^{n}$ is supported 
outside a $\eta$-neighborhood of $\Omega_{S}(t)$. Recalling that $\alpha_{n}$ is bounded in $W^{1, \infty}(0, T)^{N}, \Omega_{S}(t+h)$ is included in a $h$-neighborhood of $\Omega_{S}(t)$, so that $\mathbf{u}_{2,2, \eta}^{n}$ also vanishes in $\Omega_{S}(t+h)$. Hence, we have modified $\mathbf{u}_{2}^{n}$ into $\mathbf{u}_{2, \eta}^{n}$ which is an admissible test function on $[t, t+h]$ and satisfies

$$
\begin{gathered}
\left|\mathbf{u}_{2}^{n}-\mathbf{u}_{2, \eta}^{n}\right|_{L^{2}((0, T) \times \Omega)} \leq C \eta\left|\nabla \mathbf{u}^{n}\right|_{L^{2}((0, T) \times \Omega)}, \\
\left|\nabla \mathbf{u}_{2}^{n}\right|_{L^{2}\left(0, T ; L^{p}(\Omega)\right)} \leq C_{p} \eta^{-d(1 / 2-1 / p)}\left|\nabla \mathbf{u}^{n}\right|_{L^{2}((0, T) \times \Omega)} .
\end{gathered}
$$

The shifted velocity $\mathbf{u}_{2,+}^{n}$ is handled similarly. The estimates associated to the test function $\mathbf{w}_{2}^{n}$ are classical [2] [6]. We obtain

$$
\int_{0}^{T} \int_{\Omega}\left(\rho_{+}^{n} \mathbf{u}_{+}^{n}-\rho^{n} \mathbf{u}^{n}\right) \cdot \mathbf{w}_{2}^{n} d t d \mathbf{x} \leq C h^{\alpha_{*}}, \text { where } \alpha_{*}=\frac{2}{d+2} .
$$

We finally have to take care of the elastic velocity $\mathbf{w}_{E}^{n}:=\widehat{\mathbf{u}}_{+, E}^{n}-\widehat{\mathbf{u}}_{E}^{n}$. Using the fact that $\underline{\mathrm{x}}_{E}^{n}$ is bounded in $C^{0,1}([0, T] \times \Omega)$, we first remark that

$$
\left|\widehat{\mathbf{u}}_{E}^{n}(t, \mathbf{x})-\widehat{\mathbf{u}}_{E}^{n}\left(t, \underline{\mathrm{X}}_{E}^{n}(t, t+h, \mathbf{x})\right)\right|_{L^{2}((0, T) \times \Omega)} \leq C h,
$$

and that a similar estimate for $\widehat{\mathbf{u}}_{+, E}^{n}$ yields

$$
\begin{aligned}
& \int_{0}^{T} \int_{\Omega}\left(\rho_{+}^{n} \mathbf{u}_{+}^{n}-\rho^{n} \mathbf{u}^{n}\right) \cdot \mathbf{w}_{E}^{n} d t d \mathbf{x} \leq C h+ \\
& \left|\int_{0}^{T} d t\left(\int_{\Omega} \rho_{+}^{n} \mathbf{u}_{+}^{n} \cdot \phi_{2}^{n} d \mathbf{x}-\int_{\Omega} \rho^{n} \mathbf{u}^{n} \cdot \phi_{1}^{n} d \mathbf{x}\right)\right|,
\end{aligned}
$$

where the functions $\phi_{i}^{n}, i=1,2$, are given by

$$
\begin{aligned}
& \phi_{1}^{n}(t, \mathbf{x})=\widehat{\mathbf{u}}_{E}^{n}\left(t+h, \underline{\mathrm{X}}_{E}^{n}(t+h, t, \mathbf{x})\right)-\widehat{\mathbf{u}}_{E}^{n}(t, \mathbf{x}), \\
& \phi_{2}^{n}(t, \mathbf{x})=\widehat{\mathbf{u}}_{E}^{n}(t+h, \mathbf{x})-\widehat{\mathbf{u}}_{E}^{n}\left(t, \underline{\mathrm{x}}_{E}^{n}(t, t+h, \mathbf{x})\right) .
\end{aligned}
$$

In particular, we have

$$
\phi_{1}\left(t, \underline{\mathrm{X}}_{E}^{n}(t, 0, \mathbf{x})\right)=\phi_{2}\left(t, \underline{\widehat{X}}_{E}^{n}(t+h, 0, \mathbf{x})\right)=\psi_{0}^{h}(t, \mathbf{x}),
$$

where

$$
\psi_{0}^{h}(t, \mathbf{x})=\widehat{\mathbf{u}}_{E}^{n}\left(t+h, \underline{\widehat{X}}_{E}^{n}(t+h, 0, \mathbf{x})\right)-\widehat{\mathbf{u}}_{E}^{n}\left(t, \underline{\mathrm{X}}_{E}^{n}(t, 0, \mathbf{x})\right) .
$$


Now, we can apply the weak formulation, and end the proof of Proposition 1.

It is now easy to conclude using Kolmogorov compactness theorem that $\mathbf{u}^{n}$ converges strongly to $\mathbf{u}$ in $L^{2}((0, T) \times \Omega)^{d}$.

In order to prove that $(\rho, \mathbf{u})$ is a weak solution, we consider a test function $\phi \in \mathcal{V}$, and prove that (16) holds. As in [5] [6], we approximate $\phi$ by $\phi^{n} \in \mathcal{V}_{n}$, which converge to $\phi$ in strong topologies, then pass to the limit in $n$.

\section{References}

[1] C. Conca, J. San Martin, M. Tucsnak, Analysis of a fluid-rigid body problem, C. R. Acad. Sci. Paris Sér. I Math. 328 (1999), no. 6, p. 473-478.

[2] B. Desjardins, Global Existence Results For the Incompressible DensityDependant Navier-Stokes Equations, Differential and Integral Equations, no 3 (10) p. 587-598, 1997.

[3] B. Desjardins, Linear Transport Equations With Initial Values In Sobolev Spaces and Application to the Navier-Stokes Equations, Differential and Integral Equations, no 3 (10) p. 566-586, 1997.

[4] B. Desjardins, On weak solutions of the compressible isentropic NavierStokes equations. Applied Math. Letters, Vol 12, (7), (1999), p. 107-111.

[5] B. Desjardins, M.J. Esteban, Existence of weak solutions for the motion of rigid bodies in a viscous fluid, Arch. in Rat. Mech. Anal., 146 (1999) p. 59-71.

[6] B. Desjardins, M.J. Esteban, On weak solutions for fluid-rigid structure interaction: compressible and incompressible models, to appear in Comm. P. D. E. (1999).

[7] R.J. Di Perna, P.-L. Lions, Ordinary differential equations, transport theory and Sobolev spaces, Invent. Math. 98 (1989), p. 511-547.

[8] D. Errate, M.J. Esteban, Y. Maday, Couplage fluide-structure. Un modèle simplifié en dimension 1. C. R. Acad. Sci. Paris Sér. I Math. 318 (1994), no. 3 , p. $275-281$.

[9] C. Farhat, M. Lesoinne, P. Le Tallec, Load and motion transfer algorithms for fluid/structure interaction problems with non-matching discrete interfaces: momentum and energy conservation, optimal discretization and application to aeroelasticity. Comput. Methods Appl. Mech. Engrg. 157 (1998), no. 1-2, p. 95-114. 
[10] G.P. Galdi, J.G. Heywood, Y. Shibata, On the global existence and convergence to steady state of Navier-Stokes flow past an obstacle that is started from rest, Arch. Rational Mech. Anal. 138 (1997), no. 4, p. 307-318.

[11] C. Grandmont, Y. Maday, Analyse et méthodes numériques pour la simulation de phénomènes d'interaction fluide-structure, Actes du 29ème Congrs d'Analyse Numérique: CANum'97 (Larnas, 1997), p. 101-117 (electronic), ESAIM Proc., 3, Soc. Math. Appl. Indust., Paris, 1998.

[12] C. Grandmont, Y. Maday, Existence for a non steady fluid-structure interaction problem, Math. Model. Numer. Anal., to appear.

[13] K.-H. Hoffmann, V.N. Starovoitov, On a motion of a solid body in a viscous fluid. Two-dimensional case, Adv. Math. Sci. Appl. 9 (1999) p. 633-648.

[14] J. Leray, Essai sur les mouvements plans d'un liquide visqueux que limitent les parois, J. Math. Pures Appl. 13 (1934), p. 331-418.

[15] P. Le Tallec, J. Mouro, Fluid structure interaction with large structural displacements, Preprint.

[16] P.-L. Lions, Mathematical Topics in Fluid Mechanics, Vol 1. Incompressible models, and Vol 2, Compressible Models, Oxford Univ. Press, (1996).

[17] V. Moreau, Fluid-Structure interaction: applications in the context of a flow in arterial medium CRS4 internal report: CRS4-TECH-REP97/15 February 97

[18] A. Quarteroni, M. Tuveri, A. Veneziani, Computational Vascular Fluid Dynamics: Problems, Models and Methods, Computing and Visualisation in Science, 2000, vol 2, 163-197.

[19] A. Quarteroni, A. Veneziani, P. Zunino, Mathematical and Numerical Modelling of Solute Dynamics in Blood Flow and Arterial Walls, EPFLDMA Analyse et Analyse Numerique Report n. 12, 1999, submitted to SIAM J. Num. Analysis, 2000.

[20] D. Serre, Chute libre d'un solide dans un fluide visqueux incompressible: Existence, Japan J. Appl. Math. 4 (1987), no. 1, p. 33-73.

[21] V.A. Solonnikov, Unsteady motion of a finite mass of fluid, bounded by a free surface, J. Soviet Math. 40 (1988), p. 672-686.

[22] L. Tartar, The compensated compactness method applied to systems of conservation laws Systems of nonlinear partial differential equations (Oxford, 1982), p. 263-285, NATO Adv. Sci. Inst. Ser. C: Math. Phys. Sci., 111, Reidel, Dordrecht-Boston, Mass., 1983.

Recibido: 22 de Mayo de 2000

Revisado: 30 de Noviembre de 2000 